\title{
Prevalence et Facteurs de Risque de Lombalgie Chez Le Personnel Soignant A Lome (Togo)
}

\author{
Fianyo E., \\ Service de Rhumatologie, CHR Lomé Commune, Université de Lomé-Togo \\ Agbobli Y.A., \\ CHU Campus, Université de Lomé-Togo
}

Kakpovi K.,

Service de Rhumatologie, CHR Tomdè-Kara, Université de Kara-Togo

Houzou P.,

Service de Rhumatologie, CHU Kara, Université de Kara-Togo

Koffi-Tessio V.E.S.,

Service de Rhumatologie, CHU Sylvanus Olympio,

Université de Lomé-Togo,

Tagbor K.,

Service de Rhumatologie, Hôpital de Bè, Université de Lomé-Togo

\section{Djanda M.,}

CHR Lomé Commune, Université de Lomé-Togo

\section{Oniankitan $O$.,}

Service de Rhumatologie, CHU Sylvanus Olympio,

Université de Lomé-Togo,

\section{Mijiyawa M.,}

Service de Rhumatologie, CHU Sylvanus Olympio,

Université de Lomé-Togo

Doi:10.19044/esj.2019.v15n33p264 URL:http://dx.doi.org/10.19044/esj.2019.v15n33p264

Résumé

Objectif: Décrire la fréquence et les facteurs de risque associés à la lombalgie commune chez le personnel soignant du CHR Lomé Commune (CHR LC). Patients et Méthode: Il s'est agi d'une étude transversale réalisée du 15 décembre 2018 au 15 mars 2019 par autoquestionnaire auprès de 126 membres du personnel soignant hospitalier du CHR LC. Résultats: 79 travailleurs sur 126 ont rapporté une lombalgie au cours des 12 mois précédant l'enquête soit une prévalence de $62,7 \%$. Ils se répartissaient en 51 femmes et 28 hommes. Il s'agissait majoritairement d'infirmiers (37 cas, 29,4\%) et de gardes malades (24cas, 19\%). L'âge moyen des lombalgiques était de $42 \pm 8,1$ ans. Les facteurs de risque identifiés étaient l'âge $(\mathrm{p}=0,002)$, le sexe féminin 
$(\mathrm{p}=0,026)$, et la non connaissance des règles d'hygiène de la colonne vertébrale $(\mathrm{p}=0,034)$. La prise en charge de la lombalgie a été essentiellement symptomatique et a reposé sur les antalgiques $(88,9 \%)$, les AINS (80,5\%), et la kinésithérapie $(53,2 \%)$. Seuls 28 soignants $(26,6 \%)$ connaissaient les règles d'hygiène de la colonne. Trente-quatre soignants $(43 \%)$ avaient bénéficié d'un arrêt de travail d'une durée moyenne de 09 jours $\pm 7,5$. Le nombre total de journées de travail perdues était 281. Deux soignants $(2,5 \%)$ avaient bénéficié d'un changement de poste, et quatre soignants $(5,1 \%)$ d'un aménagement de poste (sortie du tour de garde). Conclusion: L'impact de la lombalgie sur la vie professionnelle des soignants justifie une senbilisation de ceux-ci aux facteurs de risque et aux mesures préventives.

Mots clefs: Lombalgie, Professionnels de santé, Facteurs de risque, Togo 


\title{
Prevalence and Risk Factors of Low Back Pain Among Hospital Staff in Lomé (Togo)
}

\author{
Fianyo E., \\ Service de Rhumatologie, CHR Lomé Commune, Université de Lomé-Togo \\ Agbobli Y.A., \\ CHU Campus, Université de Lomé-Togo
}

Kakpovi K.,

Service de Rhumatologie, CHR Tomdè-Kara, Université de Kara-Togo

Houzou P.,

Service de Rhumatologie, CHU Kara, Université de Kara-Togo

Koffi-Tessio V.E.S.,

Service de Rhumatologie, CHU Sylvanus Olympio,

Université de Lomé-Togo,

Tagbor K.,

Service de Rhumatologie, Hôpital de Bè, Université de Lomé-Togo

\section{Djanda M.,}

CHR Lomé Commune, Université de Lomé-Togo

\section{Oniankitan $O$.,}

Service de Rhumatologie, CHU Sylvanus Olympio,

Université de Lomé-Togo,

\section{Mijiyawa M.,}

Service de Rhumatologie, CHU Sylvanus Olympio,

Université de Lomé-Togo

\begin{abstract}
Objective: To describe the prevalence and the factors associated with low back pain among the health professionals in CHR Lomé Commune (CHR LC) in Togo. Patients and methods: A cross-sectional study was performed from December 2018 to March 2019 and included 126 staff members who filled-in a self-reported questionnaire. Results: 79 health workers $(62.7 \%)$ among the 126 hospital staff reported low back pain during the preceding twelve months. They were 51 women and 28 men. The mean age of LBP patients was $42 \pm 8.1$ years. The majority were nurses (37 cases, $29.4 \%$ ) and nurse aides (24 cases, 19\%). Their mean age was $42 \pm 8,1$ years. Factors associated with Low back pain included age $(\mathrm{p}=0.002)$, female gender $(\mathrm{p}=0.026)$, and lack of knowledge of back care techniques $(\mathrm{p}=0.034)$.
\end{abstract}


Analgesics (88.9\%), NSAID (80.5\%), and physiotherapy (53.2\%) were the most used tratements. Only 21 staff members $(26,6 \%)$ knew back care techniques. Days off duty (absenteeism) has been observed in 34 (43\%) of the hospital staff (mean : 09 days $\pm 7,5$, total number of 281 days during the period of the survey). Professional activities were restricted in 6 cases $(7,5 \%)$. Conclusion The professionnal impact of low back pain on health professionals is considerable. Risk factors and preventive measures should be taught.

Keywords: Occupationnal back pain, Prevalence, Risk factors, Health workers, Togo

\section{Introduction}

La lombalgie commune constitue un véritable problème de santé publique. Elle est le premier motif de consultation en rhumatologie (Houzou et al, 2013; INRS-France, 2018). Diverses études témoignent qu'elle constitue également un problème majeur en milieu de travail à cause des conséquences socio-professionnelles importantes et du coût élevé induit pour la société par l'absentéisme et par la consommation médicale qu'elle entraine (Kazuhiro. et al, 2001 ; Aptel et al, 2006 ; Bevan, 2015 ; Piedrahita, 2006). En milieu hospitalier, comme dans l'industrie, cette pathologie semble être fréquente et cette dernière est souvent influencée par les conditions de travail (Alcouffe et al, 2001 ; Ouedrago et al, 2010 ; Kakpovi et al, 2017). Les infirmiers sont particulièrement exposés en raison de sollicitation intensive du rachis lombaire par les mouvements de flexion et de rotation lors des soins, ainsi que la manutention de charges lourdes (Maul et al, 2003 ; Smedley et al; 1995, Marras, 2000). Cependant les autres professionnels de santé sont eux aussi touchés (Oude Hengel et al, 2011 ; Milhem et al, 2016 ; Penkala et al, 2018). Il existe une corrélation entre la qualité de vie au travail et la qualité des soins (Haute Autorité de Santé-France, 2016). Notre étude a eu pour objectif d'évaluer la prévalence et les facteurs associés à la lombalgie commune du personnel soignant du CHR Lomé Commune.

\section{Patients et Méthode}

Il s'est agi d'une étude descriptive réalisée du 15 décembre 2018 au 15 mars 2019. Elle a eu pour cadre le Centre Hospitalier Régional Lomé Commune . Ce centre dessert une population d'un million d'habitants sur une superficie de $90 \mathrm{~km}^{2}$. Il a une capacité de 126 lits, et compte 178 soignants. Le personel administratif n'a pas été inclu dans l'étude. Les 162 soignants présents à leur poste au cours de la période d'étude, après consentement éclairé, ont été soumis à un auto-questionnaire portant sur les données sociodémographiques, les antécédents médicaux, l'existence d'une lombalgie au cours des douze derniers mois, l'histoire de la lombalgie et les différents 
traitements reçus. L’ancienneté au poste désigne le nombre d'années au cours desquelles le soignant a occupé sa fonction, indépendamment du lieu d'exercice. L'hygiène de la colonne consiste à éviter la flexion du tronc lors des activités de la vie quotidienne. Cent soixante-deux questionnaires ont été distribués avec un taux de réponse de $77,8 \%$. Les tests statistiques du chi 2 ont été réalisés avec le logiciel Stata version 13.0. Une valeur de $\mathrm{p}<0,05$ a été considérée comme statistiquement significative.

\section{Résultats}

Cent vingt-six membres du personnel soignant sur un total de 178 (70, $8 \%$ ) ont rempli le questionnaire. Leurs données sociodémographiques sont résumées dans le tableau 1. Quatre soignants (3,2\%) avaient un antécédent de traumatisme lombaire, quatre soignants $(3,2 \%)$ souffraient d'hypertension artérielle, un soignant $(0,8 \%)$ souffrait de diabète, et un soignant avait une cardiopathie $(0,8 \%)$.

Soixante-dix-neuf soignants $(62,7 \%)$ avaient souffert de lombalgie au cours des douze mois précédant l'enquête. Cinquante-un $(64,5 \%)$ d'entre eux étaient de sexe féminin. Il s'agissait majoritairement d'infirmiers $(29,4 \%)$ et de gardes malades (19\%). Leur ancienneté au poste de travail était en moyenne de 12,5 $\pm 6,3$ ans (extrêmes de 1 et 30 ans). L'existence d'une lombalgie était influencée par l'âge, le sexe, et la méconnaissance des règles d'hygiène de la colonne (tableau 2).

Trente-quatre des 79 soignants lombalgiques (43\%) avaient bénéficié d'un arrêt de travail. La durée moyenne était de $09 \pm 7,5$ jours avec des extrêmes de 3 et 30 jours. Le nombre de journées de travail perdues pour lombalgie les 12 derniers mois était de 281.

Tableau 1 : Données sociodémographiques des 126 soignants interrogés

\begin{tabular}{|c|c|c|c|}
\hline & $\begin{array}{l}\text { Soignants } \\
\text { lombalgiques }\end{array}$ & $\begin{array}{l}\text { Soignants non } \\
\text { lombalgiques }\end{array}$ & $\begin{array}{l}\text { Total des } \\
\text { soignants }\end{array}$ \\
\hline Sex-ratio $(\mathrm{F} / \mathrm{H})$ & 1,82 & 0,74 & 1,2 \\
\hline Age moyen (ans) $\pm \mathrm{ET}^{*}$ & $41,3 \pm 8,2$ & $37,4 \pm 5,8$ & $39,8 \pm 7,5$ \\
\hline $\begin{array}{l}\text { Niveau d'instruction primaire ou } \\
\text { secondaire }\end{array}$ & 27 & 14 & 41 \\
\hline Niveau d'instruction universitaire & 52 & 33 & 85 \\
\hline Célibataires ou divorcés & 15 & 16 & 31 \\
\hline Mariés & 64 & 31 & 95 \\
\hline Médecins & 6 & 7 & 13 \\
\hline Paramédicaux & 73 & 40 & 113 \\
\hline Agents en poste depuis moins de 15 ans & 54 & 40 & 94 \\
\hline Agents en poste depuis plus de 15 ans & 25 & 7 & 32 \\
\hline
\end{tabular}


Tableau 2 : Facteurs favorisant la lombalgie

\begin{tabular}{cc}
\hline & Valeur de p \\
\hline Age & 0,002 \\
Catégorie professionnelle & Non \\
& significatif \\
Ancienneté dans le poste & 0,004 \\
Sexe & 0,026 \\
Statut matrimonial & Non \\
& significatif \\
Connaissance des règles d'hygiène de la & 0,034 \\
colonne & \\
\hline
\end{tabular}

Quarante-six soignants lombalgiques $(58,2 \%)$ avaient consulté un professionnel de santé. La prise en charge de la lombalgie a été essentiellement symptomatique (figure 2).

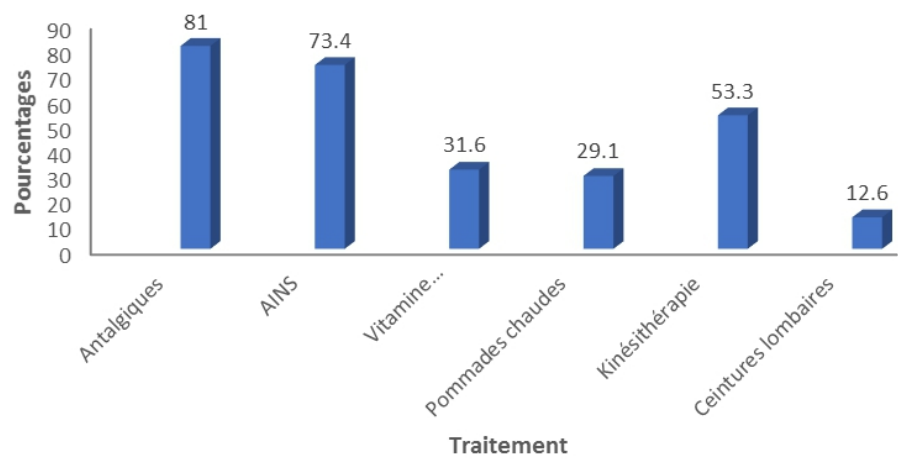

Figure 2: Répartition du personnel soignant lombalgique du CHR LC en fonction du traitement employé

Deux soignants $(2,5 \%)$ avaient bénéficié d'un changement de poste, et quatre soignants $(5,1 \%)$ avaient bénéficié d'un aménagement de poste (sortie $\mathrm{du}$ tour de garde). Cinquante-huit lombalgiques $(73,4 \%$ des soignants lombalgiques), ignoraient les règles d'hygiène de la colonne.

\section{Discussion}

Toute interprétation des données de l'étude doit tenir compte des limites du travail. L'enquête s'est limitée à rapporter les déclarations des soignants, méthode qui pourrait exposer au biais d'informations. De plus, elle est monocentrique et les résultats ne peuvent pas être généralisés à l'ensemble des soignants souffrant de lombalgie au Togo. Cependant ces biais ne sauraient modifier fondamentalement le sens de nos résultats.

Cette étude a retrouvé une fréquence de $62,7 \%$ de la lombalgie chez le personnel soignant du CHR LC, avec une nette prédominance féminine concordant avec les résultats de Ouedraogo en milieu hospitalier (Ouedrago et 
al, 2010), et les données de la littérature dans la population générale (LeResche, L. 1999, Fillingim, R.B. et al, 2009). Cette prédominance féminine résulterait de l'association de facteurs biologiques, psychologiques, et sociaux (Husky et al, 2018).

L'âge a été identifié comme un facteur de risque dans cette étude comme dans celles de Ouedraogo et Kakpovi (Ouedrago et al, 2010 ; Kakpovi et al, 2017). L'ancienneté au poste n'a été retrouvée que dans la présente étude. Il est aujourd'hui reconnu que la manutention manuelle de charges, les mouvements en avant, en arrière ou de torsion du tronc, et les vibrations transmises au corps entier sont des postures de travail qui favorisent la détérioration discale (Petit et al, 2014). En milieu hospitalier la manutention du patient reste encore souvent manuelle (Alcouffe, et al, 2001; Maul et al, 2003). Ceci est particulièrement vrai en milieu africain où l'éducation des professionnels de santé en matière d'ergonomie physique est rare, et où les équipements sont le plus souvent vétustes (Balique, 2004).

La prise en charge de la lombalgie a ainsi été essentiellement symptomatique. La durée moyenne des arrêts de travail était de $09 \pm 7,5$ jours. Ceci va à l'encontre des recommandations actuelles. Il est aujourd'hui admis que l'inactivité peut aggraver la lombalgie et en retarde le plus souvent la récupération (Direction générale Humanisation du travail- Belgique, 2008). Ainsi Abolfotouh dans une étude sur la lombalgie chez les infirmières qatariennes a trouvé une durée moyenne d'arrêt de travail de 2,03 \pm 3.09 jours (Abolfothouh SM. Et al, 2015).

Les mesures de prévention primaire et secondaire étaient quasiment absentes puisque $73,4 \%$ des soignants lombalgiques ignoraient les règles d'hygiène de la colonne. La connaissance des règles d'hygiène de la colonne était un facteur protecteur dans l'étude. C'est aussi le cas dans la littérature internationale (Tosunoz et al, 2017), à condition que les soignants mettent en pratique les bons comportements (Mwilila, 2008). La prévention de la lombalgie professionnelle repose également sur les mesures d'ergonomie physique : ajuster les hauteurs des plans de travail afin d'améliorer la posture, installer des aides mécaniques pour déplacer les charges, améliorer les surfaces de circulation afin de diminuer les frottements, les chocs et les vibrations (Direction générale Humanisation du travail- Belgique, 2008; INRS-France, 2018). Une expérience menée dans l'état de Washington a mis en évidence une baisse de $43 \%$ en quatre ans des plaintes du personnel hospitalier après mise en place d'un programme destiné à éliminer la manutention manuelle des patients (Charney et al, 2006).

\section{Conclusion}

Cette étude a mis en evidence une prevalence élevée de la lombalgie commune chez des soignants relativement jeunes, et leur totale 
méconnaissance des règles de prevention. La mise en oeuvre de programmes d'éducation des soignants et la modernination des équipements est une absolue nécessité.

\section{References:}

1. Abolfothouh, S.M., Mahmoud, K., Moammer, G., El Sayed, A., et Abolfothouh, M.A. (2015). Prevalence, consequences and predictors of low back pain among nurses in a tertiary care setting. Int Orthop; 39 : 2439-49

2. Alcouffe, J., Fabin, C., Manillier, P., Pignerol, S., Vedrenne, F., et Monteleon, P.Y. (2001). Les lombalgies chez les femmes travaillant en milieu de soins. Arch. Mal. Prof., 62 (1): 11-21

3. Aptel, M., et Gaudez, C. (2006). Affections de l'appareil locomoteur en rapport avec l'exercice d'une profession. EMC Appareil locomoteur ; 1(3):1-17

4. Balique, H. (2004). L'hôpital public en Afrique Francophone. Med Trop, 64:545-51

5. Bevan, S. (2015). Economic impact of musculoskeletal disorders (MSDs) on work in Europe. Best Pract Res Clin Rheumatol ; 29:356-73

6. Charney, W., Simmons, B., Lary, M., et Metz, S. (2006). Zero lift programs in small rural hospitals in Washington state: reducing back injuries among health care workers. AAOHN J; 54 : 355-8

7. Direction générale Humanisation du travail- Belgique. (2008). Prise en charge de la lombalgie en médecine du travail. Recommandations de bonnes pratiques. Consulté le 20 juillet 2019 sur https://docplayer.fr/11558957-Prise-en-charge-de-lalombalgie-en-medecine-du-travail.html

8. Fillingim, R.B., King, C.D., Ribeiro-Dasilva, M.C., RahimWilliams, B., et Riley, J.L. (2009). Sex, gender, and pain: a review of recent clinical and experimental findings. J Pain;10:447-85

9. Haute Autorité de Santé-France. (2016). Revue de littérature. Qualité de vie au travail et qualité des soins. Consulté le 10 juillet 2019 https://www.has-sante.fr/upload/docs/application/pdf/201602/revue_de_litterature qualite_de_vie_au travail.pdf

10. Houzou, P., Oniankitan, O., Kakpovi, K., Koffi-Tessio,V.E.S., Tagbor, K.C., Fianyo, E., et Mijiyawa, M. (2013). Profil des affections rhumatismales observées à Lomé (Togo). Tunis Méd, 91: 16-20

11. Husky, M.M., Ferdous Farin, F., Compagnone P., Fermanian, C., et Kovess-Masfety V. (2018). Health and Quality of Life Outcomes 16:195 
12. INRS-France. (2018). Travail et lombalgie. Du facteur de risque au facteur de soin. Consulté le 20 juillet 2019 http://www.inrs.fr/media.html?refINRS=ED\%206087

13. Kakpovi, K. Gblomatsi, A.S., Fianyo, E. Koffi-Tessio, V.E.S., Houzou, P., Tagbor K.C. Atassime, S., Oniankitan, O., et Mijiyawa, M. (2017). Prévalence et facteurs de risque associés à la lombalgie chez le personnel hospitalier à Lomé. J. Rech. Sci. Univ. Lomé (Togo), 19(1) : 281-290

14. Kazuhiro, K., Yasumasa, S., et Masabumi, M. (2001). An epidemiological study on occupational low back pain among people who work in construction. J Nippon Med Sch, 68: 310-17

15. LeResche, L. (1999). Gender considerations in the epidemiology of chronic pain. Epidemiology of pain;17:43-52

16. Marras, W.S. (2000). Occupational low back disorder causation and control. Ergonomics; 43:880-902

17. Maul, I., Läubli, T., Klipstein, A., et Krueger H. (2003). Course of low back pain among nurses: a longitudinal study across eight years. Occup Environ Med;60:497-503

18. Milhem, M., Kalichman, L., Ezra, D., et Alperovitch-Naienson, D. (2016). Work-related musculoskeletal disorders among physical therapists: A comprehensive narrative review. Int J Occup Med Environ Health; 29 :735-47

19. Mwilila, M.C. (2008). Work-related low back pain among clinical nurses in Tanzania [Mémoire en ligne]. University of the Western Cape. Department of Physiotherapy, Faculty of Community and Health Sciences, University of the Western Cape. Consulté le 30 septembre 2019 https://core.ac.uk/download/pdf/58913540.pdf

20. Oude Hengel, K.M., Visser, B., et Sluiter, J.K. (2011).The prevalence and incidence of musculoskeletal symptoms among hospital physicians: a systematic review. Int Arch Occup Environ Health; 84:115-9

21. Ouedraogo, D.D., Ouedraogo, V., Ouedraogo, L.T., Kinda, M., Tieno, H., et Zoungranna, E.I. (2010). Prévalence et facteur de risque associé à la lombalgie chez le personnel hospitalier à Ouagadougou. Med Trop., 70: 277-805

22. Penkala, S., El-Debal, H., et Coxon, K. (2018). Work-related musculoskeletal problems related to laboratory training in university medical science students: a cross sectional survey. BMC Public Health ; 29;18(1):1208

23. Petit, A., et Roquelaure, Y. (2014). Pathologie discale et maladie professionnelle. Rev Rhum Monographies,81:52-6 
24. Piedrahita, H. (2006). Costs of work-related musculoskeletal disorders (MSDs) in developing countries: Colombia case. Int J occup saf Ergon; 12:379-86

25. Smedley, J., Egger, P., Cooper, C., et Coggon, D. (1995). Manual handling activities and risk of low back pain in nurses. Occup Environ Med ; 52:160-3

26. Tosunoz, I.K., et Oztunc, G. (2017). Low Back Pain in Nurses. Int J Caring Sci; $10: 1728-32$ 